\title{
Evidence against global attention filters selective for absolute bar-orientation in human vision
}

\author{
Matthew Inverso $^{1}$ - Peng Sun ${ }^{1,2} \cdot$ Charles Chubb $^{1} \cdot$ Charles E. Wright $^{1} \cdot$ George Sperling $^{1}$
}

Published online: 30 October 2015

(C) The Psychonomic Society, Inc. 2015

\begin{abstract}
The finding that an item of type $A$ pops out from an array of distractors of type $B$ typically is taken to support the inference that human vision contains a neural mechanism that is activated by items of type $A$ but not by items of type $B$. Such a mechanism might be expected to yield a neural image in which items of type $A$ produce high activation and items of type $B$ low (or zero) activation. Access to such a neural image might further be expected to enable accurate estimation of the centroid of an ensemble of items of type $A$ intermixed with to-be-ignored items of type $B$. Here, it is shown that as the number of items in stimulus displays is increased, performance in estimating the centroids of horizontal (vertical) items amid vertical (horizontal) distractors degrades much more quickly and dramatically than does performance in estimating the centroids of white (black) items among black (white) distractors. Together with previous findings, these results suggest that, although human vision does possess bottom-up neural mechanisms sensitive to abrupt local changes in bar-orientation, and although human vision does possess and utilize top-down global attention filters capable of selecting multiple items of one brightness or of one color from among others, it cannot use a top-down global attention filter capable of selecting multiple bars of a given absolute orientation and filtering bars of the opposite orientation in a centroid task.
\end{abstract}

Keywords Selective attention - Visual perception .

Orientation $\cdot$ Centroid paradigm $\cdot$ Feature-based attention

Matthew Inverso

minverso@uci.edu

1 Cognitive Sciences, University of California, Irvine, Irvine, CA, USA

2 Department of Psychology, New York University, New York, NY 10003, USA

\section{Introduction}

A target bar of a given orientation pops out from a field of distractor bars of some other fixed orientation provided the difference in orientation between target and distractor bars is large enough (Beck \& Ambler, 1973; Foster \& Ward, 1991a; Foster \& Westland, 1995, 1998; Nothdurft, 1991, 1992, 1993, 1994; Sagi \& Julesz, 1985; Treisman, 1985; Treisman \& Souther, 1985; Wolfe et al., 1992). This frequently replicated result might be taken to suggest that human vision possesses a number (perhaps a continuum) of mechanisms selective for different orientations. Since Hubel and Wiesel $(1959,1962)$, it has been known that area $\mathrm{V} 1$ of occipital cortex contains neurons selective for orientation. Subsequently, Philips and Wilson (1984) measured the size and properties of the receptive fields of such neurons. Using the orientation information available in V1, a mechanism to enable orientation popout might be realized in the brain by a retinotopically organized array of neurons, each monitoring a different location of the visual field for energy in the same fixed, oriented spatial frequency band. Under this theory, a target bar would pop out from a given field of distractor bars if (at least) one of these hypothetical mechanisms were sensitive to the orientation of the target bar but not to the orientation of the distractor bars.

\section{Evidence supporting the existence of mechanisms selective} for absolute orientation

Treisman and Gormican (1988) noted that it is easier to detect an oblique bar amid vertical distractors than a vertical bar amid oblique distractors. Foster and Ward (1991a) extended this finding by making careful measurements of the threshold orientation difference required to detect a target bar amid a field of distractors as function of the orientation of the distractors. The threshold orientation difference between 
target vs. distractor bars was found to vary strongly as a function of the orientation of the background bars. In particular (in accordance with the results of Treisman \& Gormican (1988)), the orientation increment threshold was minimal when the distractor bars were either vertical or horizontal and maximal when the target bar was either vertical or horizontal.

Foster and Ward (1991a) were able to model their results in terms of two orientation-selective mechanisms that we will denote $V$ and $H$, both with broad, symmetric tuning curves, $V$ with peak sensitivity to vertical and $H$ to horizontal orientations. To understand how the model works, consider the condition in which the background bar segments are vertical and the target bar is slightly oblique from vertical. In this case, mechanism $V$ is nearly useless for the classification task, because it produces high, nearly equal responses to all bars in the display. However, mechanism $H$ is very useful in this case; although the response of $H$ to all bars in the display is much smaller than $V$ 's response, $H$ 's response to a vertical bar is substantially smaller than its response to a slightly oblique bar; thus the ratio of $H$ 's response to the target bar vs. its response the background bars is high. Under the model (elaborated in detail by Westland \& Foster, 1995), it is the relative activation produced by the target versus distractor bars rather than the difference in activation that is the crucial factor controlling performance in the classification task.

Foster and Westland (1998) investigated whether human vision possesses orientation-selective mechanisms more finely tuned than those discovered by Foster \& Ward (1991a). Foster and Westland (1998) used displays similar to those used by Foster and Ward (1991a) again measuring threshold values of the angle between a target and background bar elements as a function of background-element orientation. This time, however, they measured the angular increment threshold across a more finely sampled domain of angles: $\theta=0,5, \ldots$ 175 deg. (rather than the set $\theta=0,22.5, \ldots 167.5 \mathrm{deg}$. used by Foster \& Ward (1991a)). In addition to the two "coarse" mechanisms $V$ and $H$ discovered by Foster and Ward (1991a), this more fine-grained analysis revealed "intermediate" mechanisms with preferred orientations spaced at angles of approximately 35-50 deg. and "fine" mechanisms spaced at angles of approximately 10-25 deg. Although the two coarse mechanisms have clear peak sensitivities to the cardinal orientations (vertical and horizontal), the tuning of the intermediate and fine mechanisms tends to be more idiosyncratic across different participants.

\section{Evidence supporting the existence of mechanisms selective for orientation contrast}

The work of Foster and colleagues (Foster \& Ward, 1991a, 1991b; Foster \& Westland, 1995; Westland \& Foster, 1995; Foster \& Westland, 1998) suggests that human vision possesses an ensemble of mechanisms selective for different absolute orientations. Other research, however, both psychophysical (Nothdurft, 1991, 1992, 1993,1994; Koene \& Li, 2007; Sagi \& Julesz, 1985) and neurophysiological (Kastner, Nothdurft \& Pigarev, 1999; Nothdurft, Gallant \& Van Essen, 1999, 2000; Schofield \& Foster, 1995; Westland \& Foster, 1995; Van Essen et al., 1989) suggests that human vision possesses at least one mechanism sensitive to "orientation contrast" (as opposed to absolute orientation). Such mechanisms are hypothesized to respond not to bars of a specific, absolute orientation but rather to visual input patterns in which the predominant direction of bar orientation undergoes an abrupt change. Indeed, Nothdurft $(1992,1993,1994)$ presents evidence to support the claim that all preattentive sensitivity to orientation is conferred by mechanisms sensitive to orientation contrast (implying that human vision is devoid of mechanisms sensitive to absolute orientation).

The existence of mechanisms sensitive to orientation contrast is suggested by Fig. 1 (after Fig. 2 from Nothdurft (1994)). This image is densely populated with bars of different orientations. Throughout most of the display, the orientations of nearby bars change very gradually. However, the orientation gradient steepens abruptly at a locus of points in the image that defines a square. All bar orientations are represented in equal proportions (1) within the square, (2) outside the square, and (3) at the boundary of the square. Thus, any mechanism tuned to a particular absolute orientation would be activated by only a few bars in the image; nonetheless, the entire square emerges very clearly as a figure against the background, suggesting the existence of a mechanism that is activated by all points at the boundary of the square, i.e., by points of high orientation contrast.

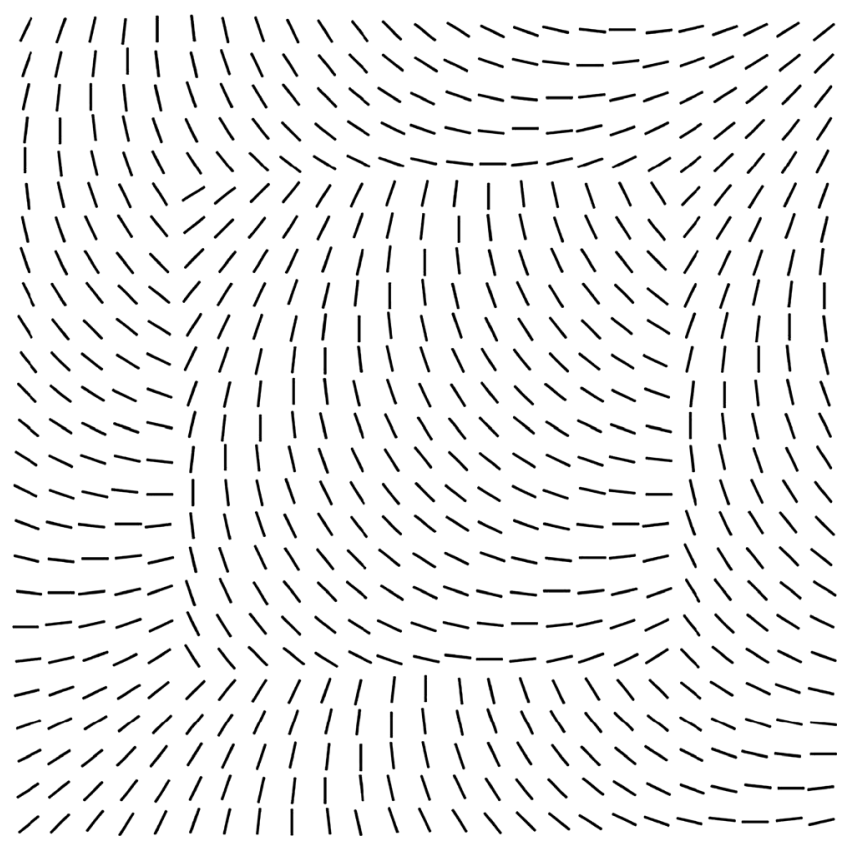

Fig. 1 (After Nothdurft, 1994). Abrupt changes in orientation suffice to produce segmentation 


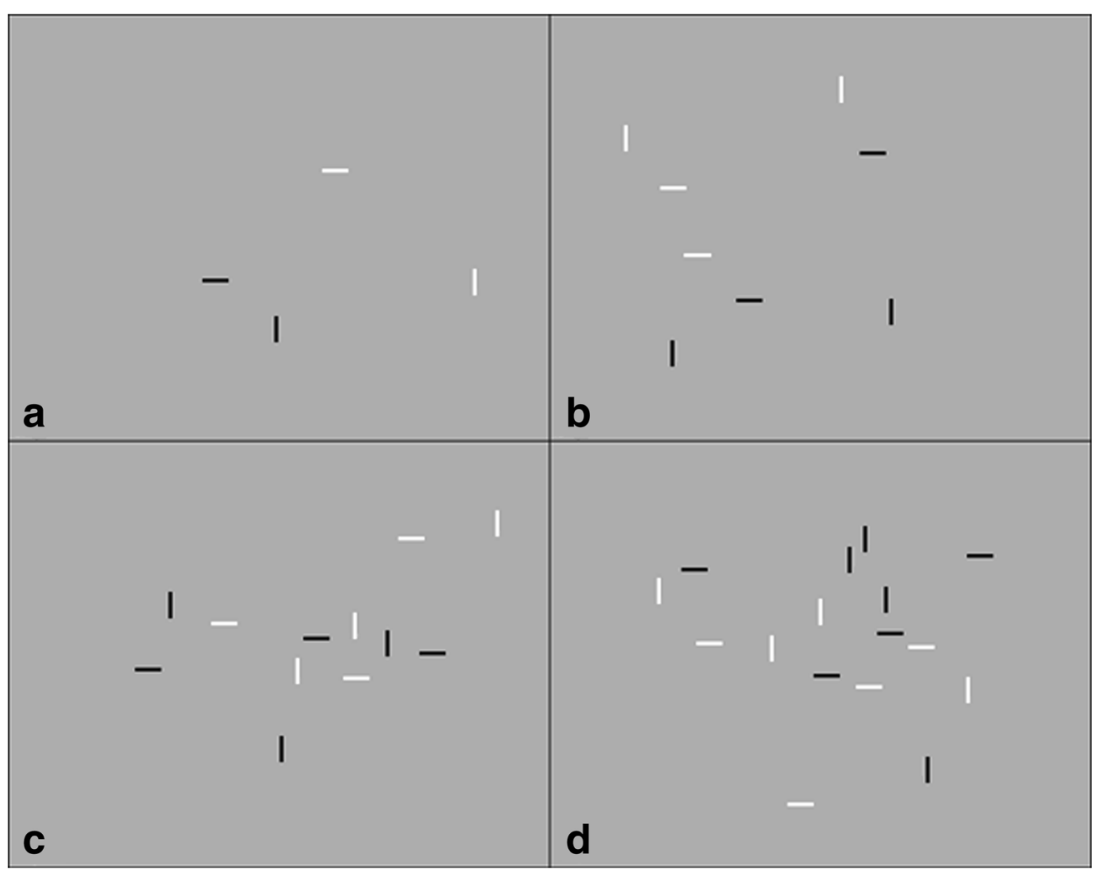

Fig. 2 Examples of stimuli from Experiment 1. (a) One token of each bar type. (b) Two of each bar type. (c) Three of each bar type. (d) Four of each bar type

\section{Current study}

Although the research of Foster and colleagues (Foster \& Ward, 1991a, 1991b; Foster \& Westland, 1995; Westland \& Foster, 1995; Foster \& Westland, 1998) seems to suggest the existence in human vision of mechanisms selective for different absolute orientations, it is important to note that Foster's experiments involve participants looking for the existence of a unique bar - one that differs from everything around it. Participants are not made aware before each trial of the orientation of the target bar, so they are not attending to any one specific orientation. As such, the Foster experiments do not demonstrate whether or not orientation-selective mechanisms are accessible in an attention task. At issue is the question: Does (top-down) selective attention in human vision have access to mechanisms sensitive to absolute bar-orientation?

The current study provides evidence that the answer to this question is no. The strategy in brief is as follows: If participants do have access to a neural image (Robson, 1980) in which, for example, vertical bars produce strong activation but horizontal bars do not, then they should be able to use this neural image to make accurate estimates of the centroids of just the vertical bars in displays comprising mixtures of vertical target bars and horizontal distractor bars. The experiments reported here demonstrate that participants are dramatically worse at this centroid-estimation task than they are if target vs. distractor items are defined by brightness (black vs. white) instead of by orientation, suggesting that although human vision possesses attention filters selective for black vs. white (Drew, Chubb \& Sperling, 2010), it does not possess corresponding attention filters selective for vertical vs. horizontal bar-orientation.

\section{Methods: experiment 1}

\section{Participants}

This study included 8 participants, 4 males and 4 females, one of whom is an author on this paper, and 3 of whom had no previous experience in centroid estimation tasks. All methods were approved by the UC Irvine Institutional Review Board, and each participant provided signed consent.

\section{Stimuli}

There were four different types of display (shown in Fig. 2). Each display contained equal numbers of horizontal black, horizontal white, vertical black and vertical white bars. For $n$ $=1,2,3,4$, " $n$-each" displays contained $n$ of each of the four different types of bars. Thus, for example, every 3 -each display contained 3 horizontal black, horizontal white, vertical black and vertical white bars-12 bars in all.

Stimuli were presented within a square region $(640 \times$ 640 pixels) subtending $20.63 \mathrm{deg}$. in width at the viewing distance of $53.5 \mathrm{~cm}$. This stimulus region was circumscribed by a thin, black frame. Each bar was $3 \times 30$ pixels in size and subtended a visual angle of 0.967 degrees in length. Note: In the center of the display, $1 \mathrm{deg}$ of visual angle equals 31.02 pixels. 
The bar positions were drawn from a circular, bivariate Gaussian distribution subject to the following three constraints: (1) There were always at least five pixels between any bars. (2) The overall spatial extent of each stimulus cloud - its Dispersion - was fixed from trial to trial. This was accomplished as follows. Let $x=\left(x_{1}, x_{2}, \ldots, x_{N}\right)$ and $y=\left(y_{1}, y_{2}, \ldots, y_{N}\right)$ be the vectors of $x$ - and $y$-coordinates of the item center locations in a given cloud, and let $\bar{X}$ and $\bar{Y}$ be the means of the vectors $x$ and $y$. Then

$$
\begin{aligned}
& \text { Dispersion }(x, y) \\
& =\left[\frac{1}{2 N-2} \sum_{i=1}^{N}\left(x_{i}-\bar{X}\right)^{2}+\left(y_{i}-\bar{Y}\right)^{2}\right]^{\frac{1}{2}} \\
& =108 \text { pixels } \approx 3.5^{\circ} .
\end{aligned}
$$

(3) The centroid of (all of the bars in) each stimulus cloud (regardless of the number of items in the display) was drawn from a circular, bivariate normal density whose mean was the center of the stimulus field and whose standard deviation subtended 41 pixels (1.28 deg of visual angle).

\section{Attention conditions}

There were four different attention conditions. In the Attendto-black condition, the participant attempted on each trial to mouse-click the centroid of all black items (all horizontal and vertical black bars) in the display, ignoring all white items. The Attend-to-white, Attend-to-horizontal, and Attend-tovertical conditions were defined analogously. Each participant participated in 16 experimental conditions, performing each of the four different attention tasks using each of the four different types of $n$-each display.

\section{Time-course of an experimental trial}

The time-course of an experimental trial is illustrated in Fig. 3. The participant first viewed a blank, gray region circumscribed by a thin black square within which each stimulus cloud would be presented. The participant initiated the first trial with a button-press; each subsequent trial followed automatically after the previous trial. After the button press, the black square disappeared and never reappeared during the trial. The remaining blank gray field was presented for $1 \mathrm{sec}-$ ond at the outset of this and each subsequent trial. It was followed by the stimulus cloud ( $300 \mathrm{~ms})$, a blank, gray screen $(33 \mathrm{~ms})$, and a random mask $(300 \mathrm{~ms})$. The random mask consisted of 100 of each of the four types of bars, arranged in a regular $20 \times 20$ grid. Pilot experiments indicated that the 333-ms SOA (Stimulus Onset Asynchrony, i.e., stimulus onset to mask onset) used was sufficient to insure that performance in all attention conditions had reached asymptotic levels. After the mask, the screen again became blank. Once the participant moved the mouse, a crosshair cursor appeared in the center of the screen and tracked the mouse movements. The participant then moved the crosshair to the remembered center of the dot cloud and entered his/her response with a mouse click. A feedback display was then presented. This display contained the original stimulus, a crosshair indicating response location entered by the participant, and a bullseye indicating the correct response location. This feedback remained visible until the participant pressed the space bar, at which point the next trial began.

\section{Design and procedure}

\section{Initial training: 1-2 sessions}

Each participant first received general training in the centroid task. This was done to (1) minimize differences in the centroid computations used by different participants and (2) decrease the noise in the responses of individual participants. Subjects without any prior centroid task experience received 800 training trials in a basic centroid task (Sun et al., 2015). Each display in this training task comprised 8 , square black dots, each subtending $0.3 \mathrm{deg}$. in width.

In addition, each participant received 800 trials of training with the specific stimuli used for this experiment, performing the task for 50 trials in each attention condition in each of the 1-each, 2-each, 3-each, and 4-each displays to become familiar with the experimental set up.

\section{Testing: 4 sessions}

Upon completing his/her training, each participant ran 16 blocks per experimental session. The attention condition was fixed across all 16 blocks in a given session. The display type (1-each, 2-each, 3-each, or 4-each) was fixed within a given block. Participants did these $n$-each blocks in order $n=1,2,3$, 4, 4, 3, 2, 1, 1, 2, 3, 4, 4, 3, 2, 1. Each block consisted of 50 trials (for participants 1, 2, 3, and 4) or 53 trials (for participants 5, 6, 7, and 8). Forty-five of these trials were "full-set" trials of the sort described above; five other "target-only" trials contained only the target bars that would occur in a full-set trial without any of the distractor bars; and (for participants 5, 6,7 , and 8 only) each block contained three additional "singleton" trials in which the display contained only a single bar which was randomly selected to be one of the two target types (e.g., either a horizontal white or a horizontal black bar in the Attend-to-horizontal condition) whose location was distributed identically to the location of the correct response on a full-set trial.

Subjects completed four such sessions - one for each of the four attention tasks. The order of these attention tasks was 


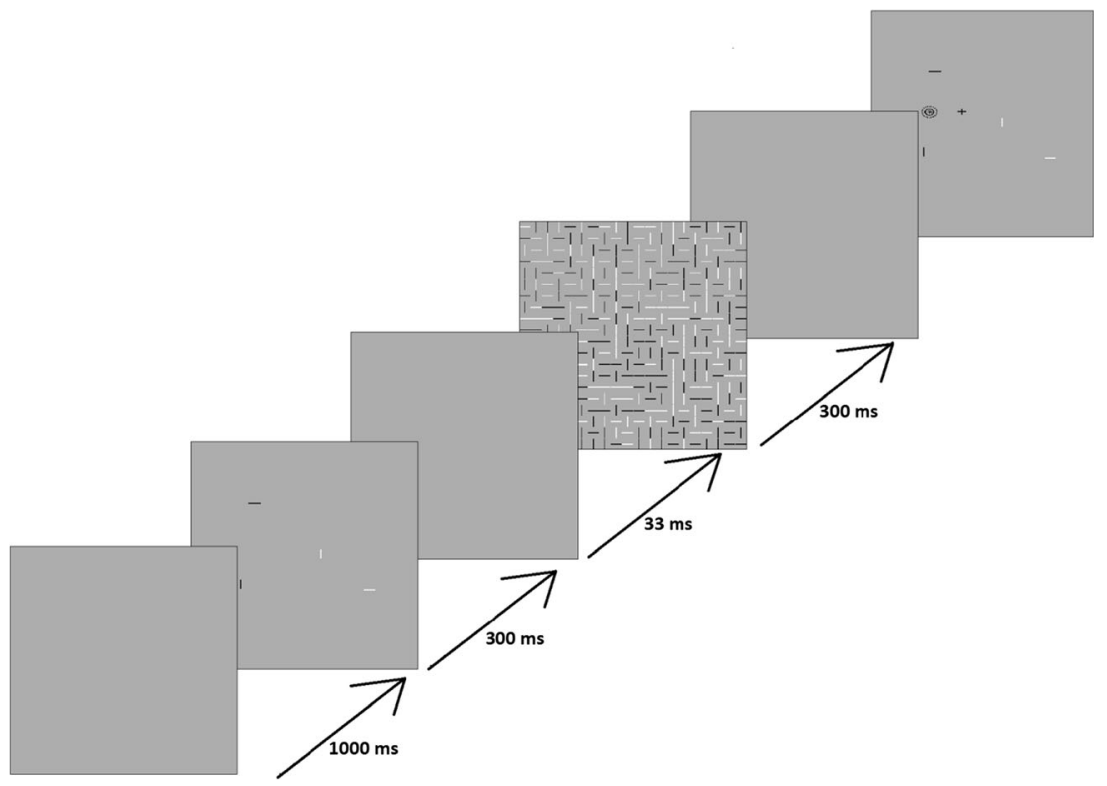

Fig. 3 Time-course of an experimental trial. Each display is presented for the full time indicated. Feedback in this trial is based on the attend-to-black condition

counterbalanced across subjects. Prior to each block, the participant was shown a gray screen containing each of the two types of target bars as well as each of the two types of distractor bars. Trials typically took approximately $2.5 \mathrm{sec}$; there was rest between blocks, and a session consisting of 800 trials took approximately $45 \mathrm{~min}$.

Participant 1 ran an additional session, in which each stimulus cloud contained 4 target bars and 16 distractor bars. This session included 16 blocks, 4 for each attention condition, and within each block there were 45 full-set trials, and 5 targetonly trials.

Data analysis We will write " $v w$," " $v b$," " $h w$," and " $h b$ " for the vertical-white, vertical-black, horizontal-white, and horizontal-black bar types. In the Attend-to-Property task (where Property is one of "black," "white," "vertical," or "horizontal"), the participant strives to click on the centroid of two target bar types and ignore the other two distractor types. (In the Attend-to-white task, for example, the target types are $v w$ and $h w$, and the distractor types are $v b$ and $h b$.) Thus, on a trial in which type $e_{i}$ is the bar type of the $i^{\text {th }}$ item in the display and $x_{i}$ and $y_{i}$ are the $x$ - and $y$-coordinates of its location, the target location $\left(\operatorname{Targ}_{\mathrm{x}}, \operatorname{Targ}_{\mathrm{y}}\right)$ is

$$
\begin{aligned}
\operatorname{Targ}_{X} & =\frac{1}{W} \sum_{i=1}^{4 n} f_{\text {targ }}\left(\text { type }_{i}\right) x_{i} \text { and } \operatorname{Targ}_{Y} \\
& =\frac{1}{W} \sum_{i=1}^{4 n} f_{\text {targ }}\left(\text { type }_{i}\right) y_{i}
\end{aligned}
$$

where $f_{\text {targ }}$ is a real-valued function of bar type that assigns equal weight to two target types and weight 0 to the two distractor types, and $W$ is the sum of $f_{\text {targ }}\left(\right.$ type $\left.e_{i}\right)$ taken over all $4 n$ items $i$ in the display.

Typically, however, the participant cannot achieve this goal; instead, the $x$ - and $y$-coordinates of his/her response $\left(R_{X}\right.$ and $R_{Y}$ ) can be well-approximated by

$R_{X}=\frac{D}{W} \sum_{i=1}^{4 n} f\left(\right.$ type $\left._{i}\right) x_{i}+(1-D) x_{\text {default }}+$ Noise $_{X}$

and

$R_{Y}=\frac{D}{W} \sum_{i=1}^{4 n} f\left(\right.$ type $\left._{i}\right) y_{i}+(1-D) y_{\text {default }}+$ Noise $_{Y}$

where $x_{\text {default }}$ and $y_{\text {default }}$ are the $x$ - and $y$-coordinates of the location toward which the participant's response is assumed to revert if he/she extracts only partial information on a given trial, $D$ is a real number between 0 and $1, f$ is a real-valued function of bar type, $W$ is the sum of $f\left(\right.$ type $\left._{i}\right)$ over all items $i$ in the display, and Noise $_{X}$ and Noise $_{Y}$ are normally distributed random variables with mean 0 and standard deviation $\sigma$.

The function $f$ is called the attention filter achieved by the participant in the given condition. The values $f(v w), f(v b)$, $f(h w)$, and $f(h b)$ are called the filter weights of the four bar types. It should be noted that $f$ is only defined up to an arbitrary multiplicative constant. For plotting purposes, we impose the constraint that $f(v w)+f(v b)+f(h w)+f(h b)=1$.

The parameter $D$ is called the Data-drivenness of participant's response. If $D=1$, then the participant's response on a given trial is determined exclusively by the items in the stimulus cloud. At the other extreme, if $D=0$, then the participant's response on a given trial is 
influenced not at all by the stimulus but instead by the combined effects of random noise plus a tendency to click on the fixed location $\left(x_{\text {default }}, y_{\text {default }}\right)$.

\section{Estimating model parameters}

To estimate the model parameters $x_{\text {default }}, y_{\text {default }}, f, D$ and $\sigma$, we proceed as follows:

1. Let $L_{X}\left(L_{Y}\right)$ be the $N_{\text {trials }} \times 6$ matrix whose $(i, j)^{\text {th }}$ entry is the sum of the $x$-locations ( $y$-locations) of all items of type $e_{j}$ presented on trial $i$ for $j<5$, is $1(0)$ for $j=5$, and is 0 (1) for $j=6$.

2. Let $R_{X}\left(R_{Y}\right)$ be the column vector of length $N_{\text {trials }}$ whose $i^{\text {th }}$ entry is the $x$-coordinate ( $y$-coordinate) of the participant's response on trial $i$.

Then

1. Form the $2 N_{\text {trials }} \times 6$ matrix $M$ by appending the matrix $L_{Y}$ to the bottom of $L_{X}$.

2. Form the vector $R$ of length $2 N_{\text {trials }}$ by appending $R_{Y}$ to $R_{X}$.

3. Perform linear regression to derive the weights $W$ minimizing $S S_{\text {Residual }}=\|M W-R\|^{2}$.

Then, writing $n_{j}, j=1,2,3,4$, for the number of items of type $j$ in each stimulus cloud in a given condition, $D$ (Datadrivenness) is estimated by

$D=\sum_{j=1}^{4} W_{j} n_{j}$

For $j=1,2,3,4, f(j)$ is estimated by

$f(i)=\frac{W_{j}}{\sum_{i=1}^{4} W_{i}}$

$\sigma^{2}$ is estimated by taking

$\sigma^{2}=\frac{S S_{\text {residual }}}{d f}, \quad d f=2 N-6$

(where model parameters $x_{\text {default }}, y_{\text {default }}$, and $D$ absorb 3 degrees of freedom and the attention filter $f$ absorbs only 3 additional degrees of freedom, because it is constrained to sum to 1 ). Finally, $x_{\text {default }}$ and $y_{\text {default }}$ are estimated by taking

$x_{\text {default }}=\frac{W(5)}{1-D}$ and $y_{\text {default }}=\frac{W(6)}{1-D}$

A formal justification of this modeling method and a description of the methods used to estimate confidence intervals for model parameters is provided in Sun et al. (2015).

\section{Quantifying performance}

Note that actual performance (as described by Eq. (2)) can deviate from target performance (Eq. (1)) for several reasons. We quantify these deviations using:

1. Imperfect Data-drivenness. The Data-drivenness $D$ of the participant's responses can be less than 1 .

2. Filter mismatch. The attention filter $f$ achieved by the participant can deviate from the target filter $f_{\text {targ }}$. This will cause the responses of the participant to deviate systematically from the correct responses. To quantify the degree to which the responses of the participant are immune from this sort of error, we use two descriptors of the participant's attention filter $f$. The first, called the Selectivity, is a ratio - the participant's attention weight for targets divided by the attention weight for distracters. Selectivity is given by

Selectivity $=\frac{f\left(t_{1}\right)+f\left(t_{2}\right)}{\left|f\left(d_{1}\right)\right|+\left|f\left(d_{2}\right)\right|}$

where $t_{1}$ and $t_{2}\left(d_{1}\right.$ and $\left.d_{2}\right)$ are the two item-types designated as targets (distractors) in the given attention condition; i.e., $f_{\text {targ }}\left(t_{1}\right)=f_{\text {targ }}\left(t_{2}\right)=0.5$, and $f_{\text {targ }}\left(d_{1}\right)=f_{\text {targ }}\left(d_{2}\right)$ $=0$. When the attention filter achieved by a participant in a given condition closely approximates the target filter, Selectivity becomes very large. For this reason, it is convenient to plot $\log _{10}$ (Selectivity) rather than Selectivity.

The second, which we call Filter-fidelity, is a measure of how far away from the ideal filter $(\phi)$ the achieved filter $(f)$ is. This is compared to how far away the worst possible filter a participant can get $\left(f_{\text {Worst }}\right)$ is from the ideal filter. Specifically, we obtain Filter-fidelity with the formula

Fidelity $=1-\frac{\|f-\Phi\|}{\left\|f_{\text {Worst }}-\Phi\right\|}$

Notice that when the participant perfectly matches this target filter, this will be 1 , and when the participant perfectly matches the worst possible filter, this will be 0 .

3. Random noise. The standard deviation $\sigma$ of the random variables Noise $_{X}$ and Noise $_{Y}$ is nonzero. This will cause the responses of the participant to deviate randomly from the correct responses. Although $\sigma$ itself could be used to gauge the amount of random error corrupting the participant's responses, this model parameter is difficult to interpret, because it depends on several factors (such as the size of stimulus display clouds) that are likely to vary across different experiments. To facilitate comparison of results across experiments, we use a descriptor called Efficiency to quantify immunity to random error. Efficiency is the greatest lower bound on the proportion of display 
items that the participant must be using to compute his/her centroid estimates. If the trial-to-trial random errors Noise $_{X}$ and Noise $_{Y}$ were due solely to missing (i.e., failing to include) some of the items in the display in computing the centroid, then the participant would need to miss a proportion $p=1-$ Efficiency of the items on each trial in order for Noise $_{X}$ and Noise $_{Y}$ to have standard deviation $\sigma$. Thus, if the participant were to attain an Efficiency of 0.75 , this would imply that he/she is including, on average, in his/her centroid computation at least three-quarters of the items in the stimulus display. It should be emphasized, however, that if some of the random noise corrupting responses were due to some other source, such as (1) early perceptual noise, or (2) instability in the centroid computation, or (3) motor noise, than the actual proportion of items included in computing the centroid would be higher than Efficiency.

\section{Results: experiment 1}

Figure 4 plots the 16 attention filters achieved in each of the four attention tasks for each of the 1-each, 2-each, 3-each, and 4-each displays, across all participants. Although some participants had more experience with centroid tasks than others, there were no substantial differences between these groups. As such, all participants were pulled together for these analyses. With the 1each display, they achieve an attention filter very similar to the target filter as witnessed by the high values of Selectivity and Filter-fidelity for all four attention conditions (Fig. 5). In addition, they achieve very high Efficiencies in all four attention conditions as well as high Data-drivenness values.

With each of the 2-, 3-, and 4-each displays, however, the picture changes. Although the participants continue to perform very well in the Attend-to-white and Attend-to-black conditions, performance is dramatically impaired in the Attend-to-vertical and Attend-to-horizontal conditions. In particular, the attention filters in the Attend-to-vertical and Attend-to-horizontal conditions show very little Selectivity and Filter-fidelity for the target items vs. the distractor items; in each of these two attention conditions, the participant gives roughly equal weight to all items in the stimulus cloud, yielding values of Selectivity and Filter-fidelity that are much lower for the Attend-to-vertical and Attend-to-horizontal conditions than for the Attend-to-black and Attend-to-white conditions. Note also that with the 2-, 3-, and 4-each displays, Efficiency values are lower for the Attend-to-vertical and Attend-tohorizontal conditions than they are for the Attend-to-white and Attend-to-black conditions.

The difference in the effectiveness of attention filters for brightness vs. for orientation is dramatized by the upper two panels in Fig. 5. The left upper panel plots mean Efficiency as a function of the number of each type of item in the stimulus

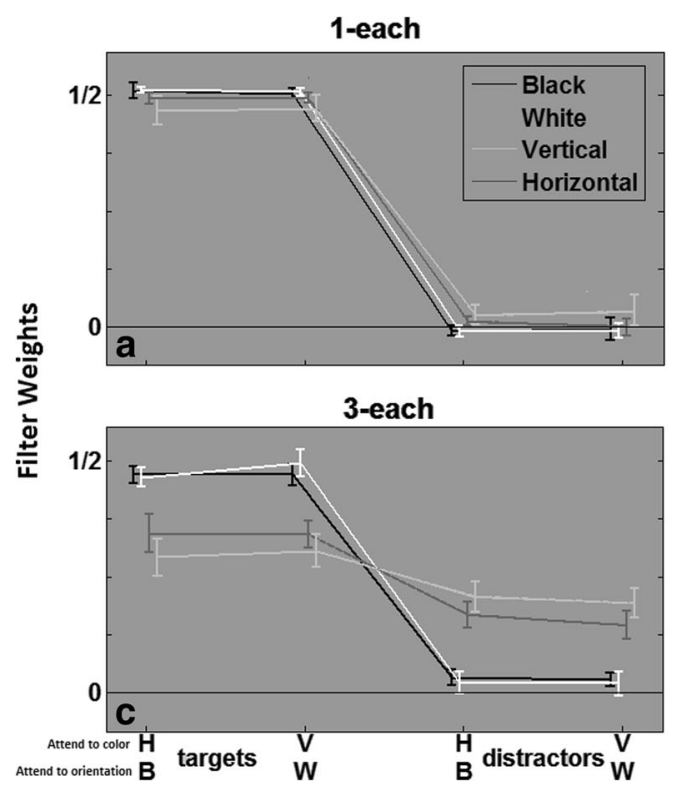

Fig. 4 Average attention filters and attention descriptors for all eight participants. Each attention filter is plotted so that its responses to the two target items are plotted on the left and its responses to the two distractor items are plotted on the right. The H's and V's labeling abscissa tick marks indicate the orientations (Horizontal or Vertical) of the targets and distractors for the Attend-to-black attention filter (plotted in black) and Attend-to-white attention filter (plotted in white). The B's

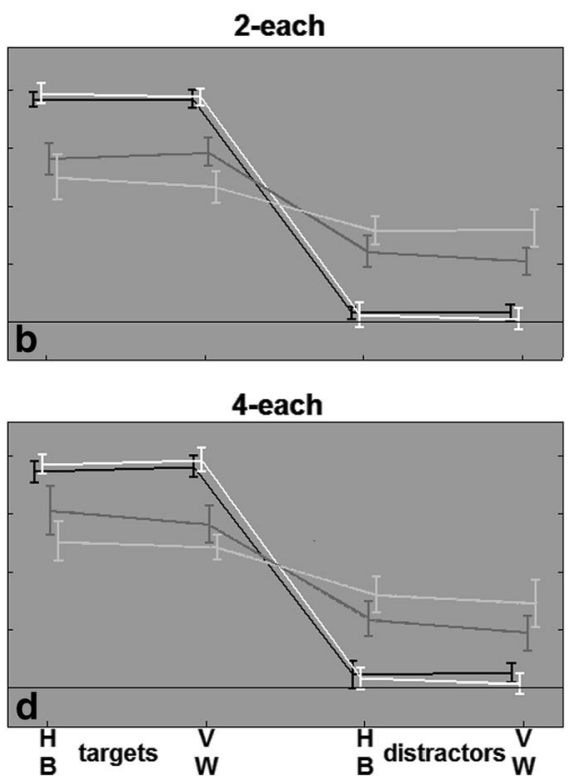

and W's indicate the color (black or white) of the targets and distractors for the Attend-to-vertical (dashed dark gray) and Attend-to-horizontal (dashed light gray) conditions. Error bars are $95 \%$ confidence intervals. Performance is excellent in all attention conditions with 1-each displays and remains excellent in Attend-to-black and -white conditions with 2-, 3, and 4-each displays but is much worse in Attend-to-horizontal and vertical conditions with 2-each, 3-each, and 4-each displays 

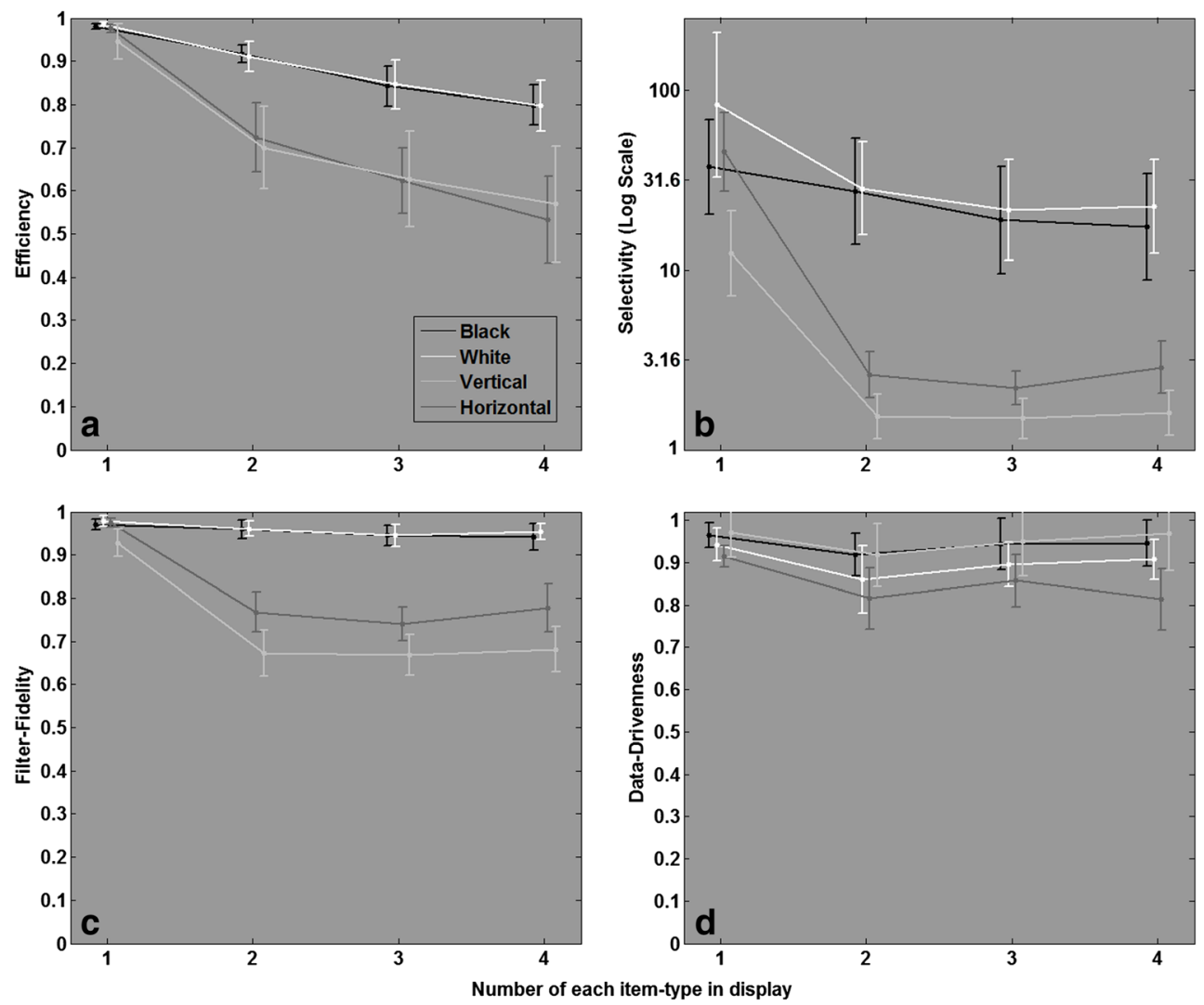

Fig. 5 Attention filter descriptors as a function of the number of items in stimulus displays for the four attention conditions. These panels plot mean Efficiency (a), mean Selectivity (b), mean Filterfidelity (c), and mean Data-drivenness (d) as a function of the number of each type of item in the stimulus display. Attention descriptor values for Attend-to-white, Attend-to-black, Attend-to-horizontal, and Attendto-vertical conditions are plotted in white, black, light gray, and dark gray,

display. The right upper panel plots the Selectivities in the same way, and the lower left and right panels show Filterfidelity and Data-drivenness, respectively. Results for Attend-to-white, Attend-to-black, Attend-to-horizontal, and Attend-to-vertical conditions are plotted in white, black, dashed light gray, and dashed dark gray respectively. The values of Efficiency, Selectivity, and Filter-fidelity are similarly high in all attention conditions for the 1-each displays; however, the values of all three filter descriptors are much lower for the Attend-to-vertical and Attend-to-horizontal conditions than for the Attend-to-black and Attend-to-white conditions for all of the 2-each, 3-each, and 4-each displays.

This observation is supported by Greenhous-Geisser corrected $\mathrm{F}$ tests. To ensure homogeneity of variance, Filterfidelities were arcsine-transformed prior to the analysis. There is a clear interaction between attention condition and displaytype $(\mathrm{F}(2.196,15.371)=57.132, p<0.001)$. However, this interaction is not seen when we restrict display types to 2each, 3-each, and 4-each $(\mathrm{F}(1.966,13.759)=1.694, p=$ 0.22 ), nor is there a strongly significant difference between attention conditions for the 1 -each displays $(\mathrm{F}(1,7)=7.168$, respectively. Error bars are $95 \%$ confidence intervals for the mean over the 8 participants. Note that all three filter descriptors take high values for all attention conditions with the 1-each displays; however, Efficiency, Filter-fidelity, and Selectivity but not Data-drivenness are much lower in the Attend-to-vertical and Attend-to-horizontal conditions than in the Attend-to-black and Attend-to-white conditions for the 2-each, 3-each, and 4-each displays

$p=0.032$ ). The mildly significant difference seen there is due strictly to the lower Filter-fidelities achieved by participants in the Attend-to-horizontal condition vs. the other three attention conditions. These results suggest that the interaction reflects mostly the difference between the 1-each vs. the 2-each, 3each, and 4-each conditions, where performance in the Attend-to-horizontal and Attend-to-vertical conditions drops off suddenly compared the Attend-to-white and Attend-toblack conditions.

Interestingly, Data-drivenness does not show an analogous pattern; the curves for the Attend-to-vertical and Attend-tohorizontal conditions do not separate clearly from those for the Attend-to-white and Attend-to-black conditions. Rather Data-drivenness values remain high in all attention conditions across all displays.

Although the difference between the Filter-fidelities achieved in the Attend-to-vertical vs. Attend-to-horizontal conditions seems small, it is statistically highly significant. Indeed, every participant in every $n$-each condition achieved higher Filter-fidelity in the Attend-to-vertical than in the Attend-tohorizontal condition. As this observation suggests, within any 
$n$-each condition, a two-tailed $t$-test confirms a significant difference between the Filter-fidelity achieved in the Attend-tovertical vs. the Attend-to-horizontal condition. (For 1-each, $t(7)$ $=4.0545, p=0.0048$. For 2-each, $t(7)=5.1402, p=0.0014$. For 3 -each $t(7)=3.7373, p=0.0072$. For 4-each, $t(7)=5.3660, p=$ 0.0010 .) Interestingly, the current results give us no reason to suppose that this effect depends on the number of items in the display; specifically, an $F$-test failed to reject the null hypothesis that the mean difference between vertical and horizontal Filterfidelities was equal across $n$-each conditions $(\mathrm{F}(3,28)=1.8859$, $p=0.1549$ ).

The current results provide no traction in understanding this effect. We speculate, however, that the vertical-vs.-horizontal advantage may be related to the vertical gravitational frame within which the current experiments were conducted. As shown by Mikellidou et al. (2015), the gravitational frame exerts a powerful influence on the "oblique effect" (i.e., the heightened sensitivity of participants to slight deviations in orientation from cardinal orientations $\left(0^{\circ}\right.$ and $\left.90^{\circ}\right)$ vs. from other orientations). If the gravitational frame operates slightly more strongly to accentuate the salience of vertical vs. horizontal bars, this could produce effects of the sort observed in Experiment 1.

\section{Discussion: experiment 1}

Attention-condition $\times$ number-of-item interaction The results document a dramatic interaction between attention condition and the number of items in the display. Performance in all of the Attend-to-white, -black, -vertical, and -horizontal conditions is at ceiling for the 1-each displays but degrades dramatically in Attend-to-vertical and Attend-to-horizontal conditions compared with the Attend-to-white and Attendto-black conditions for the 2-each, 3-each, and 4-each displays.

These results suggest that:

1. Human vision does not possess a global attention filter selective for vertical (horizontal) vs. horizontal (vertical) bar orientation. If human vision did possess such a global attention filter, then participants would be able to perform well in the centroid task in the Attend-to-vertical (Attendto-horizontal) condition irrespective of the number of items in the display. Therefore, the fact that participants are dramatically impaired in the centroid task in the Attend-to-vertical and Attend-to-horizontal conditions compared to the Attend-to-white and Attend-to-black conditions for the 2-each, 3-each, and 4-each displays suggests that, although human vision possesses a global attention filter selective for white (black) vs. black (white), it does not possess a global attention filter selective for vertical (horizontal) vs. horizontal (vertical) bar orientation.

2. Participants must have alternative strategies available for performing the centroid task in the Attend-to-vertical (Attend-to-horizontal) condition with 1-each displays. The current results reveal little about these strategies other than that they do not require access to a global attention mechanism; however, substantial previous research suggests that human vision has special capabilities for processing a small number of items (Cowan, 2001; Luck \& Vogel, 1997; Pylyshyn \& Storm, 1988; Trick \& Pylyshyn, 1993, 1994).

There are a couple alternative accounts of the results of Experiment 1 that need to be ruled out. Both of these accounts propose that human vision actually does possess global attention filters for vertical and also for horizontal orientation; however, Experiment 1 fails to reveal them.

The first account proposes that the operation of these glob$\mathrm{al}$, orientation-selective, attention filters is disrupted in Experiment 1 by the very strong variations in brightness across which the filters are required to pool. Experiment 2 addresses this possibility by using stimulus displays in which all bars vary only in the task-relevant dimension.

The second account proposes that global attention filters for horizontal and vertical orientation in human vision exist but do not admit effective binding of item orientation with item location (which is required for centroid estimation). Experiment 3 addresses this possibility by using a task that requires participants to integrate information about feature identity across space but not to bind feature identities to locations. Specifically, in this task participants are asked to judge which of two item-types in a given stimulus display (e.g., vertical vs. horizontal bars) is more numerous.

\section{Methods: experiment 2}

Experiment 2 had four participants ( 2 males, 2 females), all of whom also participated in Experiment 1.

Stimuli were generated in exactly the same fashion as in Experiment 1. However, in this case, there was no variation in the unattended feature dimension (Fig. 6). As in Experiment 1, participants saw blocks consisting of 2 targets, 4 targets, 6 targets, or 8 targets, and an equal number of distractors. Participants completed 16 conditions ( 4 attention conditions by 4 numerosity conditions), each consisting of 2 blocks of 53 trials each, defined analogously to Experiment 1 . Within any given block, each item in every display took the same fixed value on the irrelevant feature dimension. For example, in one block in the Attend-to-vertical condition, all bars (whether vertical or horizontal) in the stimulus presented on every trial 


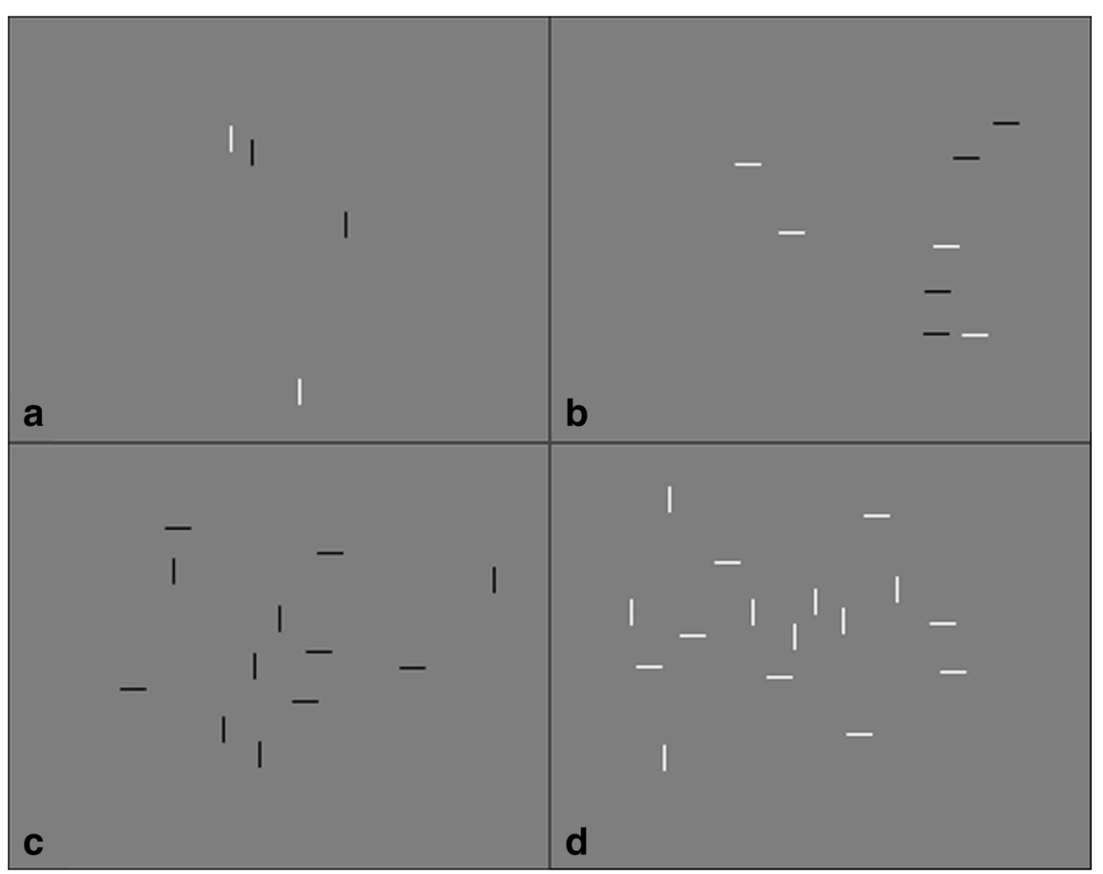

Fig. 6 Example stimuli for Experiment 2. (a) Two target bars (attending to brightness). (b) Four target bars (attending to brightness). (c) Six target bars (attending to orientation). (d) Eight target bars (attending to orientation)

were black (as shown in Fig. 6c for a 6-target display condition).

\section{Results: experiment 2}

Figure 7 plots the 16 attention filters achieved in the four attention tasks for all of the 2-target, 4-target, 6-target, and 8 -target displays, for each participant. It should be noted that Participant 1 (plotted in black below) had much more practice with centroid tasks than the other three. As in Experiment 1, with the 2-target display, all participants achieve attention filters that are very similar to the target filter. With 2-target displays, Selectivity is high for all four attention conditions, as is Efficiency (Fig. 8).

Once again, however, with each of the 4-, 6-, and 8-target displays, things change. The pattern seen in Experiment 1 remains clear. Participants can still perform very well in all conditions with only two targets, but as soon as the number of targets increases, performance in the Attend-to-vertical and Attend-to-horizontal conditions drops dramatically.

The difference in the effectiveness of attention filters for brightness versus for orientation is dramatized by Fig. 8 . The top-left (top-right, bottom) panel plots mean Efficiency (Filter-fidelity, Selectivity) as a function of the number of each type of item in the stimulus display. Results for Attend-towhite, Attend-to-black, Attend-to-horizontal, and Attend-tovertical conditions are plotted in white, black, light gray, and dark gray, respectively. The values of Efficiency, Filter-fidelity, and Selectivity are similarly high in all attention conditions for the 2-target displays; however, the values of all three filter descriptors are much lower for the Attend-to-vertical and Attend-to-horizontal conditions than for the Attend-to-black and Attend-to-white conditions for all of the 4-target, 6-target, and 8-target displays. It should be noted that Data-drivenness remains high in all four attention conditions, regardless of the number of targets. Specifically, it had a mean value of 0.92 , and ranged from 0.89 to 0.96 with no clear difference between conditions and number of targets.

$F$-tests (Greenhouse-Geisser corrected) confirm that the results of Experiment 2 follow the same general pattern as those of Experiment 1. To insure homogeneity of variance, tests were performed on arcsine-transformed Filter-Fidelities. A significant (but weak) interaction is observed between attention condition and display-type $(\mathrm{F}(1.040,3.120)=13.068, p=0.034)$. However, this interaction is not seen when display-types are restricted to 4-target, 6-target, and 8-target $(\mathrm{F}(1.030,3.091)=$ $0.834, p=0.431)$. There is a significant difference between Attention conditions in for 2-target displays $(F(1,3)=$ $111.922, p=0.002)$, primarily due to the lower Filterfidelities achieved in the Attend-to-horizontal condition vs. other conditions. The interaction again seems to reflect mostly the difference between the 2-target vs. 4-, 6-, and 8-target conditions, in which performance in the Attend-to-horizontal and Attend-to-vertical conditions drops off suddenly compared with the Attend-to-white and Attend-to-black conditions. 


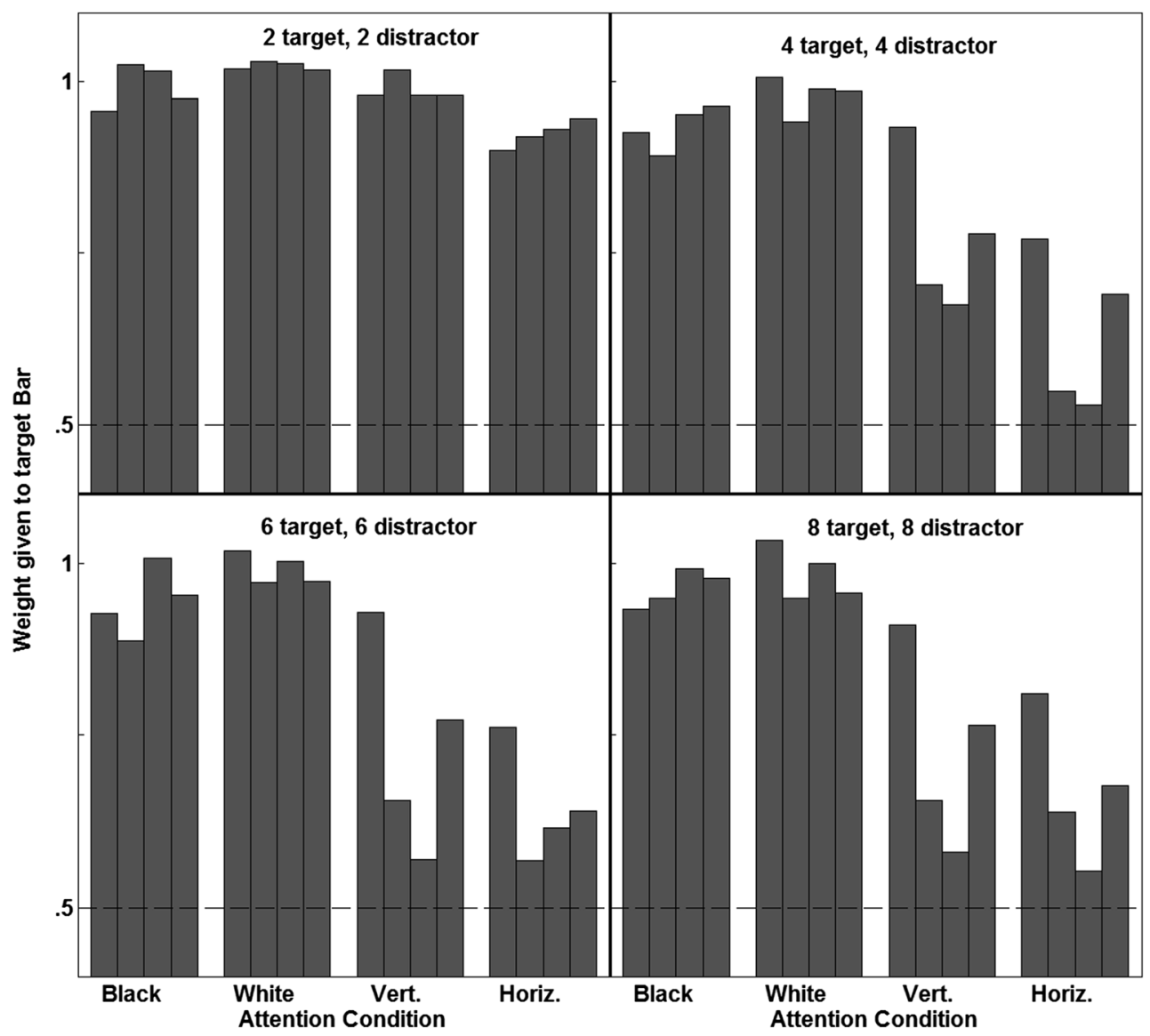

Fig. 7 Weight given to target bars for each attention and number of targets condition. Each panel shows the proportion of weight a participant gave to the target bars (compared to distractor bars), plotted for each individual participant. Performance is excellent for all display

numerosities for attention to black or white, and in the 2-target displays for attention to bar-orientation (a). In 4-target (b), 6-target (c), and 8-target (d) displays, attention filters for bar-orientation are much closer to chance performance

\section{Discussion: experiment 2}

The pattern of results is similar to that seen in Experiment 1. Performance is strong in all of the Attend-to-black, -white, vertical, and -horizontal conditions for displays containing only two target items but quickly falls to a floor in Attendto-vertical and Attend-to-horizontal conditions when the number of targets increases. Thus, in Experiment 1, the degradation in performance with 2-, 3-, and 4-each displays in the Attend-to-horizontal and -vertical conditions compared with the Attend-to-white and -black conditions cannot be attributed solely to disruption of global orientation-selective filters by irrelevant variation in brightness between items.

With that said, however, it should be noted that participant 1 (black lines in Fig. 7) showed little degradation in performance with increasing display numerosity in either of the Attend-tohorizontal or Attend-to-vertical conditions. This participant (the first author) was highly practiced in many variants of the Attend-to-vertical and Attend-to-horizontal task conditions. By contrast, none of the other participants showed improvement in Experiment 2 compared to Experiment 1 (Fig. 9).
When the number of targets is four or more, attending to orientation is much harder than attending to brightness, as in Experiment 1. This effect is somewhat weaker than we observed in Experiment 1, indicating perhaps that non-homogenous targets and/or distractors was making the task harder. However, because the effect remains regardless, this added difficulty is not what was causing the observed asymmetry between luminosity and orientation.

\section{Methods: experiment 3}

\section{Participants}

This study used three subjects ( 1 male and 2 females), one of whom is an author of this paper. All methods were approved by the UC Irvine Institutional Review Board, and each participant provided signed consent. 

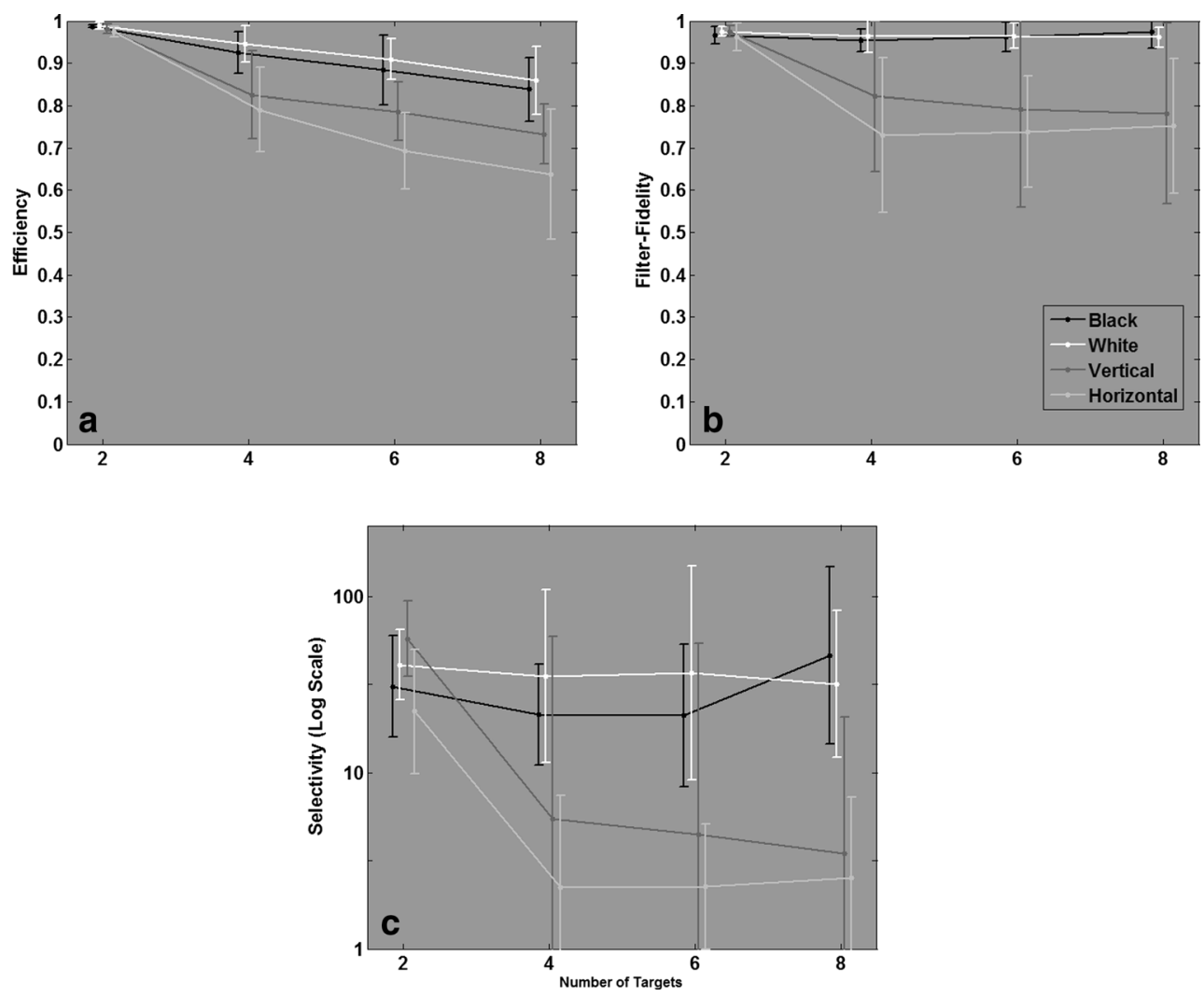

Fig. 8 Attention filter descriptors as a function of the number of targets in stimulus displays for the four attention conditions. These panels plot mean Efficiency (a), mean Filter-fidelity (b), and mean Selectivity (c) as a function of the number of targets in the stimulus display. Attention descriptor values for Attend-to-white, Attend-to-black, Attend-to-horizontal, and Attend-to-vertical conditions are plotted in white, black, dark gray, and light gray, respectively. Error bars are $95 \%$

\section{Task}

The participant was instructed to attend to one feature dimension and indicate (by keypress) which feature within that dimension was more numerous. If the feature dimension was brightness, then the participant had to judge whether there were more black bars or more white bars. If the feature dimension was orientation, then he or she had to judge whether there were more vertical bars or horizontal bars.

\section{Stimuli}

The stimuli in Experiment 3 were similar to those in Experiments 1 and 2. Once again, there were four types of bars: black-vertical, black-horizontal, white-vertical, and white-horizontal. The locations of bars within displays were subject to the same constraints as in Experiments 1 and 2. However, in the current experiment, bars of different types were not presented in equal numbers. In the Brightness-difference condition, there were equal numbers of horizontal and vertical bars ( 8 of confidence intervals for the mean over the four participants. Note that all three filter descriptors take high values for all attention conditions with the 2-target displays; however, they all are much lower in the Attend-tovertical and Attend-to-horizontal conditions than in the Attend-to-black and Attend-to-white conditions for the 4-target, 6-target, and 8-target displays

each), but unequal numbers of black and white bars (9 of one, 7 of the other). In the Orientation-difference condition, there were equal numbers of black and white bars, but unequal numbers of vertical and horizontal bars (9 of one, 7 of the other). Figure 10 shows an example stimulus from the Brightness-difference condition.

\section{Design}

Participants came in for 1 session of 8 blocks, each consisting of 60 trials. Task condition (Brightness-difference vs. Orientation-difference) was varied by block, with Participants 1 and 3 doing Brightness-Orientation-Orientation-BrightnessBrightness-Orientation-Orientation-Brightness and Participant 2 doing the opposite. All participants were trained with 60 trials in each task prior to data collection.

Each trial began with one second of a gray screen, before the stimulus appeared for $300 \mathrm{~ms}$. The screen then went blank for another $33 \mathrm{~ms}$, before a mask appeared for $300 \mathrm{~ms}$. Finally, the participant was able to respond and was given feedback. 


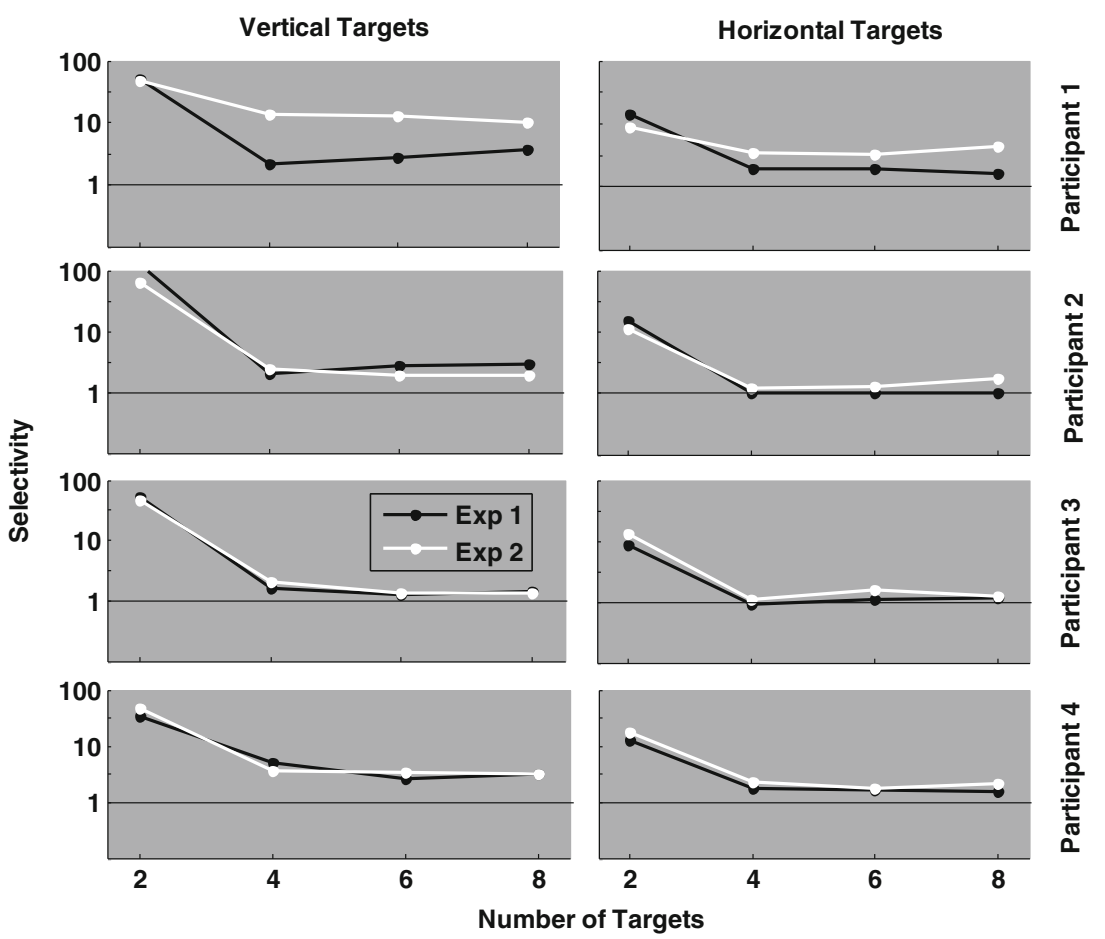

Fig. 9 Comparison of individual participants' performance (as reflected by Selectivity) in Experiment 1 vs. Experiment 2, when attending to orientation. Black lines show participants' Selectivities in Experiment 1, and white lines show their Selectivities in Experiment 2. Left plots show each participant's Selectivity when attending to vertical

The participant then initiated the next trial by pressing any key.

\section{Results: experiment 3}

Figure 11 shows the results for each of the three participants. Each participant achieves significantly higher D-prime in the Brightness-difference condition than in the Orientationdifference condition.

\section{Discussion: experiment 3}

Previous research has made it clear that people are able to estimate the numerosity of a cloud of dots of one color amid distractor dots of another color (Halberda et al., 2006). Furthermore, people can simultaneously estimate the numerosities of two intermixed clouds of dots differing in color (Poltoratski \& Xu, 2013). Thus, it is not surprising that participants achieved high dprime values in the brightness-difference condition. What is most striking about the current results is the relative deficit in performance in the orientationdifference condition. bars, and right plots show the same participant's Selectivity when attending to horizontal bars. Trends in the two experiments are the same, and with the exception of Participant 1, everyone performed just as well in Experiment 1 as in Experiment 2

Even though the task in Experiment 3 did not require the participant to bind feature-identities to locations in the visual field, every participant continued to perform better in the Brightness-difference than in the Orientation-difference condition. We conclude that the difficulty experienced by our participants in the Attend-to-horizontal and Attend-to-vertical conditions in Experiment 1 was not due to the requirement (implicit in the centroid task) that information about item orientation be bound to item locations.

It is important to note that participants did achieve dprime values well above chance even when asked about orientation. One plausible reason for this is that

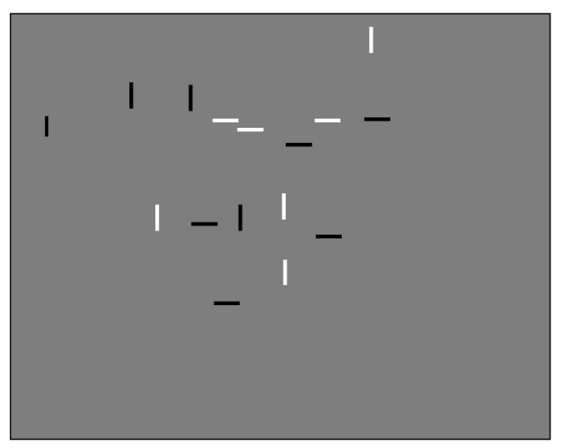

Fig. 10 Example stimulus from the Brightness-difference condition. In this task, the participant strives to judge whether the black or the white bars are more numerous (In this example, the correct answer is BLACK) 


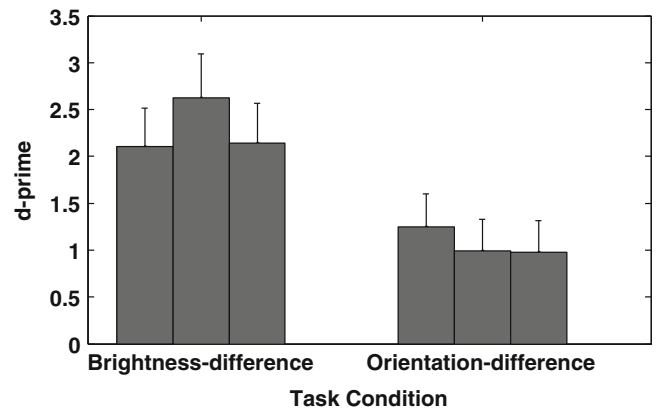

Fig. 11 Performance for each of three participants in Experiment 3 as a function of task condition. A value of d-prime equal to 0 indicates chance performance. Error bars are $95 \%$ credible intervals derived from a Markov chain Monte Carlo simulation

participants were able to extract some small subset of the bars and make their judgements based on the numerosity of that subset. According to simulations done on that assumption, subsets would need to contain between 5 and 10 bars (out of 16 total) to achieve a dprime in the range we saw in the orientation conditions. If a similar strategy were being used for brightness, 14 to 15 bars would need to be seen. This suggests people are more efficient at extracting brightness information than orientation information.

\section{General discussion}

\section{Orientation contrast mechanism}

Previous research has documented an asymmetry in the sensitivity of feature-based attention for orientation versus color (Huang, 2015; Wolfe et al., 1995). The current results support these findings and suggest a possible explanation for all of these effects. Suppose, as proposed by Nothdurft (1992, 1993, 1994), that all human preattentive sensitivity to orientation is conferred by a mechanism that is selective for orientation contrast but indifferent to absolute orientation. In Experiments 1 and 2, vertical and horizontal bars occur in equal numbers; this imbues vertical and horizontal bars with equal orientation contrast implying that performance should be very poor in the centroid task. ${ }^{1}$

Note, however, that if the number of distractors is increased relative to the number of targets, then performance in the

\footnotetext{
${ }^{1}$ The horizontal and vertical bars in an $n$-each display produce indistinguishable responses in a mechanism selective for orientation contrast for the following reason. In one of the $n$-each displays used in the current experiment, every bar, whether horizontal or vertical, shares the stimulus space with $n-1$ bars of the same orientation and $n$ bars of the opposite orientation. Thus, on average, the expected orientation contrast relative to its context of any bar in an $n$-each display is the same. For this reason, a mechanism selective for orientation contrast is useless for purposes of estimating the centroid of the target bars of one orientation in an $n$-each display while ignoring the distractor bars of the opposite orientation.
}

centroid task should improve for the following reason: because distractor bars are more numerous, target bars will tend to differ more strongly in orientation from the bars that surround them than will distractor bars. In this case, the target bars will tend to produce higher activation than distractor bars in the orientation-contrast mechanism that we hypothesize is used for the task.

\section{If participants do not have access to attention filters tuned to absolute orientation, how do they achieve better-than-chance performance in the Attend-to-horizontal and Attend-to-vertical conditions of Exp. 1?}

It is clear from Fig. 5b that participants achieve Selectivities significantly greater than 1 . (None of the confidence intervals for the Attend-to-vertical or -horizontal conditions contains 1 for any of the 1-, 2-, 3-, or 4-each displays.) One possible explanation is the following. Regardless of whether a participant can access an attention filter tuned to a specific bar orientation, he or she may be able to locate and identify several items of the target orientation in any given stimulus. If he/she gives these few items enhanced weight in his/her centroid computation, Selectivity will be elevated above 1. However, to the extent that he/she allows the unidentified items in the display to influence performance, his/her Selectivity will be suppressed. Such a strategy would plausibly yield the low, but above-1 Selectivities observed in Experiment 1.

\section{$V$ and $H$ mechanisms revisited}

The broadly tuned mechanisms $V$ and $H$ with peak sensitivities to vertical and horizontal orientations hypothesized by Foster and colleagues (Foster \& Ward, 1991a, 1991b; Foster \& Westland, 1995; Westland \& Foster, 1995; Foster \& Westland, 1998) should be ideally suited for the Attend-tovertical and Attend-to-horizontal centroid task conditions of Experiments 1 and 2, as well as for the attend-to-orientation condition of Experiment 3. The $V(H)$ mechanism should be strongly activated by vertical (horizontal) bars and very weakly activated by horizontal (vertical) bars. Thus, the finding that performance is so poor in the Attend-to-vertical and Attendto-horizontal conditions with each of the 2-each, 3-each, and 4-each displays suggests that these mechanisms cannot be utilized in a global selective attention task.

\section{Absolute orientation vs. orientation contrast}

It has been argued (Friedman-Hill \& Wolfe, 1995) that people do have sensitivity to absolute orientation in a search task. However, we do not find the empirical evidence for this claim 
compelling. ${ }^{2}$ Although human vision has mechanisms that enable global selection of items based on contrast, color, shape, and other attributes, we find that human vision possesses no mechanism that enables selection of multiple items based on absolute bar-orientation. A broader range of tasks is needed to further explore the inability of observers to access mechanisms sensitive to absolute orientation. We conjecture that human vision discards information about absolute orientation because in the natural world, local orientation depends on both object and head position and is therefore not a sufficiently useful indicator to prompt the evolution of attention filters for absolute orientation. On the other hand, human vision may have evolved a preattentive mechanism sensitive to orientation contrast; this reflects the fact that orientation contrast is independent of absolute orientation and often indicates something worth noticing.

\section{Summary}

Participants were tested in a task requiring them to use topdown selective attention to estimate the centroids of target items with one feature value while ignoring distractor items with the opposite feature value. Each display contained equal numbers of white horizontal bars, white vertical bars, black horizontal bars, and black vertical bars. In the Attend-to-black (Attend-to-white) condition, the participant strove to mouseclick the centroid of all the white (black) target bars in the

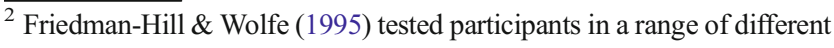
search tasks, two of which are relevant for the current discussion. In their "conjunction search" task, participants judged whether a vertical-red target bar was present among horizontal-red and vertical-green distractor bars. In their "subset search" task, the target was a red bar of orientation $\mathrm{X}^{\circ}$ among green bars of orientation $\mathrm{X}^{\circ}$ and red bars of orientation $\mathrm{Y}^{\circ}$. The orientations, $\mathrm{X}^{\circ}$ and $\mathrm{Y}^{\circ}$, were randomly chosen subject to the constraint that they had to differ by at least $30^{\circ}$. The subset search task thus forced participants to adopt a strategy of searching for a bar with an oddball orientation within the cohort of red bars. Mean reaction time in the subset search condition was significantly slower than in the conjunction search condition. The authors inferred from this result that participants have access to a different (and more effective) strategy in the conjunction task than searching within the red bars for an oddball orientation. Specifically, they suggest that in the conjunction task the participant is able to recruit spatially parallel processes selective for each of (1) bar redness and (2) bar verticality to converge on the target. Note, in particular, that this interpretation suggests the existence of a spatially parallel mechanism selective for absolute bar orientation.

We contend, however, that these findings admit other interpretations. The stimuli used in the subset task are intrinsically more complicated than those used in the conjunction task. Most importantly (in contrast to the conjunction task in which the target bar always differed from same-color distractor bars by $90^{\circ}$ ), in the subset search task the difference in orientation between the target bar and same-color distractor bars varies randomly (in both sign and magnitude) from trial to trial; moreover, this orientation difference is usually substantially less than $90^{\circ}$. If indeed the only orientation-sensitive mechanism available to participants is sensitive not to absolute orientation but rather to orientation contrast, then this disparity between the two tasks would be highly likely to impair performance selectively in the subset task vs. the conjunction task.
}

display while ignoring the black (white) distractor bars. In the Attend-to-vertical (Attend-to-horizontal) condition, the participant strove to mouse-click the centroid of all the vertical (horizontal) target bars in the display while ignoring the horizontal (vertical) distractor bars. Each participant was tested in each attention condition with displays that contained 1-each, 2-each, 3-each, or 4-each of the four bar-types.

With 1-each displays participants performed very well in all attention conditions. However, with 2-each, 3-each, and 4each displays, performance was greatly impaired in the Attend-to-vertical and Attend-to-horizontal conditions compared to the Attend-to-white and Attend-to-black conditions.

The different pattern of results observed with the 1-each displays suggests that this variant of the task (which required participants to locate the two target bars and click on the midpoint between them) afforded strategies that were not available with the 2-each, 3-each, and 4-each displays.

The fact that the Attend-to-white condition (analogous remarks apply to the Attend-to-black condition) yielded excellent performance with each of the 2-each, 3-each, and 4-each displays suggests that in this condition (1) participants were able to access an attention filter that produced high activation in response to white target bars and low (or zero) activation to black distractor bars, and (2) the output of this mechanism provided a neural image from which the centroid of the target bars could be extracted. The dramatic impairment in performance observed in the Attend-to-horizontal and Attend-tovertical conditions with each of the 2-each, 3-each, and 4each displays suggests that no such strategy was available to globally select targets of one orientation from distractors of a perpendicular orientation. This suggests that participants do not have access to an attention filter that produces high activation in response to target bars of a given orientation and low (or zero) activation to distractor bars of the opposite orientation.

Additional control experiments demonstrated that the impairment of performance in the attend-to-horizontal and attend-to-vertical conditions (compared to the attend-to-black and attend-to-white conditions) persisted (1) if the stimulus displays used in the attend-tohorizontal and attend-to-vertical conditions of the centroid task were composed of items that all had the same brightness, and (2) in the context of a task (requiring the participant to judge which of two item types was more numerous in the stimulus display) in which the participant was not required to bind information about item-type to locations in the visual field.

The current results thus suggest that the high levels of performance typically achieved in tasks based on differences in orientation are mediated not by topdown mechanisms sensitive to absolute orientation but rather by a bottom-up mechanism that senses local differences in orientation. 


\section{References}

Beck, J., \& Ambler, B. (1973). The effects of concentrated and distributed attention on peripheral acuity. Perception \& Psychophysics, 14, 225-230.

Cowan, N. (2001). Metatheory of storage capacity limits. Behavioral and Brain Sciences, 24(01), 154-176.

Drew, S. A., Chubb, C. F., \& Sperling, G. (2010). Precise attention filters for Weber contrast derived from centroid estimations. Journal of Vision, (10):20, 1-16.

Foster, D. H., \& Ward, P. A. (1991a). Asymmetries in oriented-line detection indicate two orthogonal filters in early vision. Proceedings of the Royal Society of London B, 243, 75-81.

Foster, D. H., \& Ward, P. A. (1991b). Horizontal-vertical filters in early vision predict anomalous line-orientation identification frequencies. Proceedings of the Royal Society of London B, 243, 83-86.

Foster, D. H., \& Westland, S. (1995). Orientation contrast vs. orientation in line-target detection. Vision Research, 35, 733-738.

Foster, D. H., \& Westland, S. (1998). Multiple groups of orientationselective visual mechanisms underlying rapid orientated-line detection. Proceedings of the Royal Society of London B, 265, 16051613.

Friedman-Hill, S. R., \& Wolfe, J. M. (1995). Second-order parallel processing: Visual search for the odd item in a subset. Journal of Experimental Psychology. Human Perception and Performance, 21(3), 531-551.

Halberda, J., Sires, S. F., \& Feigenson, L. (2006). Multiple spatially overlapping sets can be enumerated in parallel. Psychological Science, 17(7), 572-576.

Huang, L. (2015). Color is processed less efficiently than orientation in change detection but more efficiently in visual search. Psychological Science, 26(5), 646-652.

Hubel, D. H., \& Wiesel, T. N. (1959). Receptive fields of single neurons in the cat's striate cortex. The Journal of Physiology, 148, 574-591.

Hubel, D. H., \& Wiesel, T. N. (1962). Receptive fields, binocular interaction, and functional architecture in the cat's visual cortex. The Journal of Physiology, 160(1), 106-156.

Kastner, S., Nothdurft, H. C., \& Pigarev, I. N. (1999). Neuronal responses to orientation and motion contrast in cat striate cortex. Visual Neuroscience, 16, 587-600.

Koene, A.R. \& Li, Z. (2007). Feature-specific interactions in salience from combined feature contrasts: Evidence for a bottom-up saliency map in V1. Journal of Vision 7(7):6, 1-14. http://journalofvision. org/7/7/6/, doi:10.1167/7.7.6.

Luck, S. J., \& Vogel, E. K. (1997). The capacity of visual working memory for features and conjunctions. Nature, 390(6657), 279-281.

Mikellidou, K., Cicchini, G. M., Thompson, P. G., \& Burr, D. C. (2015). The oblique effect is both allocentric and egocentric. Journal of Vision, 15(8), 24-24.

Nothdurft, H. C. (1991). Texture segmentation and pop-out from orientation contrast. Vision Research, 31, 1073-1078.

Nothdurft, H. C. (1992). Feature analysis and the role of similarity in preattentive vision. Perception \& Psychophysics, 52, 355-375.

Nothdurft, H. C. (1993). The role of features in preattentive vision: Comparison of orientation, motion and color cues. Vision Research, 33, 1937-1958.

Nothdurft, H. C. (1994). Common properties of visual segmentation. In G. R. Bock \& J. A. Goode (Eds.), Higher order processing in the visual system (Ciba Foundation Symposium 184, pp. 245-259). Chichester, UK: Wiley.
Nothdurft, H. C., Gallant, J. L., \& van Essen, D. C. (1999). Response modulation by texture surround in primate area V1: Correlates of "popout" under anesthesia. Visual Neuroscience, 16, 15-34.

Nothdurft, H. C., Gallant, J. L., \& van Essen, D. C. (2000). Response profiles to texture border patterns in area V1. Visual Neuroscience, $17,421-436$

Philips, G., \& Wilson, H. (1984). Orientation bandwidths of spatial mechanisms measured by masking. Journal of the Optical Society of America. A, 1(2), 226-232.

Poltoratski, S., \& Xu, Y. (2013). The association of color memory and the enumeration of multiple spatially overlapping sets. Journal of Vision, 13(8), 6.

Pylyshyn, Z. W., \& Storm, R. W. (1988). Tracking multiple independent targets: Evidence for a parallel tracking mechanism. Spatial Vision, 3(3), 179-197.

Robson, J. G. (1980). Neural images: The physiological basis of spatial vision. In C. S. Harris (Ed.), Visual coding and adaptability (pp. 177-214). Hillsdale, NJ: Erlbaum.

Sagi, D., \& Julesz, B. (1985). 'Where' and 'what' in vision. Science, 228, $1217-1219$.

Schofield, A. J., \& Foster, D. H. (1995). Artificial neural networks simulating visual texture segmentation and target detection in lineelement images. Philosophical Transactions of the Royal Society of London, Series B, 350(1334), 401-412.

Sun P, Chubb C, Wright CE, Sperling G. (2015). The centroid paradigm: Quantifying feature-based attention in terms of attention filters. Attention, Perception, and Psychophysics. doi:10.3758/s13414015-0978-2.

Treisman, A. (1985). Preattentive processing in vision. Computer Vision, Graphics, and Image Processing, 31(2), 156-177.

Treisman, A., \& Gormican, S. (1988). Feature analysis in early vision: Evidence from search asymmetries. Psychological Review, 95(1), $15-48$.

Treisman, A., \& Souther, J. (1985). Search asymmetry: A diagnostic for preattentive processing of separable features. Journal of Experimental Psychology: General, 114, 285-310.

Trick, L. M., \& Pylyshyn, Z. W. (1993). What enumeration studies can show us about spatial attention: Evidence for limited capacity preattentive processing. Journal of Experimental Psychology: Human Perception and Performance, 19(2), 331.

Trick, L. M., \& Pylyshyn, Z. W. (1994). Why are small and large numbers enumerated differently? A limited-capacity preattentive stage in vision. Psychological Review, 101(1), 80.

Van Essen, D. C., DeYoe, E. A., Olavarria, J., Knierim, J., Fox, J., Sagi, D., \& Julesz, B. (1989). Neural responses to static and moving texture patterns in visual cortex of the Macaque monkey. In D. M. K. Lam \& C. D. Gilbert (Eds.), Neural mechanisms of visual perception (pp. 137-154). Woodlands, TX: Portfolio.

Westland, S., \& Foster, D. H. (1995). Optimized model of oriented-linetarget detection using vertical and horizontal filters. Journal of the Optical Society of America. A, 12, 1617-1622.

Wolfe, J. M., Chun, M. M., \& Friedman-Hill, S. R. (1995). Making use of texton gradients: Visual search and perceptual grouping exploit the same parallel processes in different ways. In T. Papathomas, C. Chubb, A. Gorea, \& E. Kowler (Eds.), Early vision and beyond (pp. 189-198). Cambridge, MA: MIT Press.

Wolfe, J. M., Friedman-Hill, S. R., Stuart, M. I., \& O'Connell, K. M. (1992). The role of categorization in visual search for orientation. Journal of Experimental Psychology: Human Perception and Performance, 18, 34-49. 